\title{
Acute opioid detoxification revealing a unique challenge in clinical management
}

\author{
Alexander Sabre, Lydia Ehlenberger
}

\begin{abstract}
Introduction: Opioid abuse in pregnancy is inherit to a multitude of adverse outcomes for both mother and fetus, with use of intra-dermal injections for drug delivery leading to systemic sequela such as endocarditis. In patients with history of drug abuse, infective endocarditis most frequently presents as a subacute disease process that requires high clinical suspicion. Case Report: We report a case of a 21-year-old caucasian female, G3Poo2o, who presented to the emergency department in a state of acute opioid withdraw. Initial investigation detailed a positive pregnancy test and leukocytosis. The extensive workup revealing a viable embryo and culture negative endocarditis (CNE) with vegetation on tricuspid valve and further care ensuring proper treatment for inpatient course. Conclusion: We present a clinical case of intravenous-opioid derived CNE composed of challenges that required multiple disciplines and collaboration of multiple specialties in the gravid patient along with detailed information on management in this subset of patients.
\end{abstract}

Keywords: Endocarditis, Pregnancy, Opioid dependency

Alexander Sabre ${ }^{1}$, Lydia Ehlenberger ${ }^{2}$

Affiliations: ${ }^{1}$ Universidad Autonoma de Guadalajara-Medical Student, Guadalajara, Jalisco, Mexico; ${ }^{2}$ Pediatrix/Obstetrix Medical Group, Scottsdale Healthcare System, Department of OB/GYN Phoenix, Arizona.

Corresponding Author: Alexander Michael Sabre 2045 S McClintock Dr Apt \#233, Tempe, Arizona 85282, USA; Ph: 602-703-7563, 610-746-9642; Email: alexandermsabre@ gmail.com

Received: 26 November 2014

Accepted: 19 February 2015

Published: 23 March 2015

\section{How to cite this article}

Sabre A, Ehlenberger L. Acute opioid detoxification revealing a unique challenge in clinical management. Case Rep Int 2015;4:1-5.

Article ID: 100006CRINTAS2015

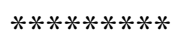

doi:10.5348/crint-2015-6-CR-1

\section{INTRODUCTION}

The first successful treatment of bacterial endocarditis in the gravid patient was described by Nazarian et al. in 1976 [1]. The incidence of infective endocarditis during pregnancy as noted in literature, is $0.006 \%$. Significant mortality for both mother and fetus has been established for this disease, with maternal mortality rate reaching $33 \%$ and fetal mortality at $29 \%$ [1].

Perinatal mortality is a significant challenge in the care of drug users. Fetal activity and oxygen demand increase during accidental withdrawal and intentional abstinence, which may coincide to hypoxic dysfunction of the placenta or labor; resulting in fetal death [2]. Ensuring that acute withdrawal from drug intoxication was minimal, placement of methadone maintenance treatment (MMT) has long been used to treat dependence in pregnancy. Although MMT can be associated with neonatal abstinence syndrome (NAS) in $60-90 \%$ of infants exposed to methadone in utero, the adverse effects of uncontrolled withdrawal highlight its practical use [3]. This standard of care endorsed by the American Academy of Pediatrics (AAP) provides many benefits which include increased fetal growth, improvements in prenatal care, and a reduction in fetal mortality [4].

We wish to present a clinical case of intravenousopioid derived CNE encountered in obstetric practice, 
provide relevant background information on opioid abuse and withdrawal in pregnancy, present the appropriate use of MMT in the pregnant patient, and to provide clinicians information on how to properly recognize, diagnose, and treat $\mathrm{CNE}$ in this patient population.

\section{CASE REPORT}

A 21-year old caucasian female $\mathrm{G}_{3}$ Poo20 presented to the emergency department with anxiety, weakness, insomnia, nausea, diarrhea, and cramps of less than 24 hours duration. The patient reports the use of a significant amount of heroin by injection daily. She reported that she wanted to undergo detox and presented to the hospital in order to "get clean".

Past medical history is significant for recreational drug abuse for nine years, asthma, and an early first trimester miscarriage at age 20. Past surgical history is significant for an abortive dilation and curettage at age 12. The patient did not take medications and had no known drug allergies. Social history revealed the patient is a current everyday smoker of half a pack of cigarettes a day, and revealed an extensive history recreational drug abuse with methamphetamine, and venous injection of 1 gram of heroin daily.

Vitals signs were blood pressure 98/50, pulse $78 /$ $\mathrm{min}$, respiratory rate $22 / \mathrm{min}$, temperature $38.4^{\circ} \mathrm{C}$, room air $\mathrm{O}_{2}$ sats 98. Physical examination revealed a young disheveled woman wrapped in a blanket in acute distress, diaphoretic, shivering, and with bilateral erythematous needle-point marks on her arms and legs. The patient was admitted for acute opioid detoxification with further evaluation and treatment.

Laboratory workup revealed a urine drug screen positive for amphetamines and heroin. A urine pregnancy screen was positive, which was followed by serum quantitative beta human chorionic gonadotropin (BHCG) with a level of $38347 \mathrm{IU} / \mathrm{L}$. Blood work was significant for extensive leukocytosis with a white blood cell (WBC) count of of $33.2 \times 10^{3} / \mu \mathrm{L}$. Initial treatment protocol was lorazepam $2 \mathrm{mg}$ for anxiety, methadone titration started at $10 \mathrm{~g}$, a nicotine patch for tobacco abuse, and social services consultation for psychosocial factors.

Investigation first began by performing a pelvic ultrasound due to the elevated BHCG levels. Ultrasound examination revealed a seven-week embryo with positive fetal heart tones (FHT), a uterus of appropriate size for first trimester time period, and normal ovaries.

Investigation into the leukocytosis and with patient's recent and extensive history of intravenous drug abuse prompted a 2d trans-thoracic echocardiogram (TTE), which revealed a $3.6 \mathrm{~mm}$ vegetative lesion on the tricuspid valve (Figure 1). There was mild mitral regurgitation, and ejection fraction was estimated at 60-65\%. Blood cultures taken peripherally from different sites did not give rise to causative organism. A diagnosis of culture negative endocarditis (CNE) prompted empirical antibiotic treatment.

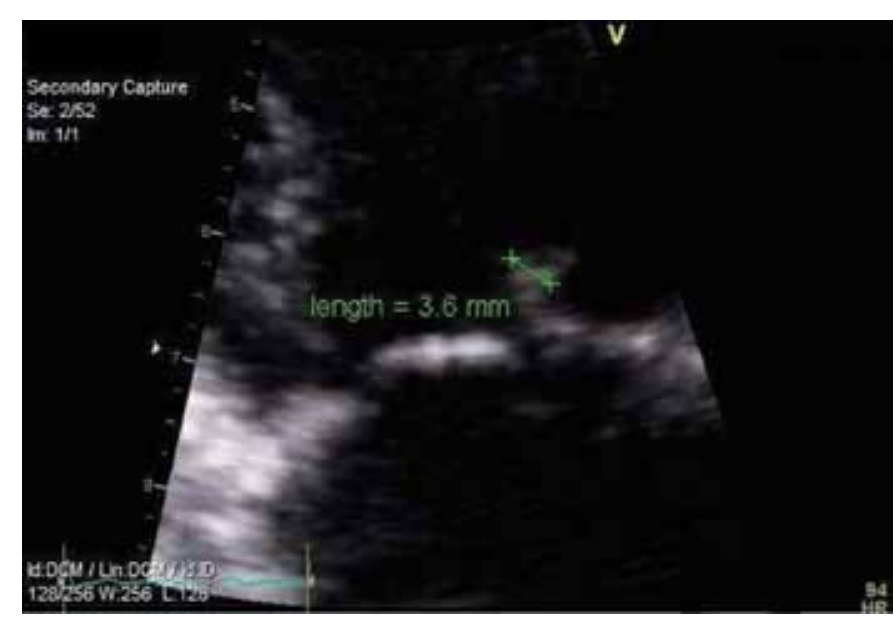

Figure 1: Transthoracic echocardiography of patient displaying the $3.6 \mathrm{~mm}$ vegetation on tricuspid valve.

Infectious disease initiated IV antibiotics consisting of IV ceftriaxone and IV vancomycin both of which were continued for 4-6 weeks. By second day, the patient WBC count dropped to $12 \times 10^{3} / \mu \mathrm{L}$ and stayed at this range for the rest of hospital stay. The patient was compliant to MMT which began with $10 \mathrm{~g}$ and decreasing to stable titration level during hospital stay. The patient was also compliant to addition of folate supplements and improved nutritional directives for the pregnancy.

The patient was followed by obstetrics/gynecology, internal medicine, and infectious disease, with the pregnancy considered viable throughout the time in hospital. After the initial presentation of acute detoxification, the patient remained afebrile and did not have any incidences of acute withdrawal symptoms for remainder of stay. The titration of MMT was decreased from initial $10 \mathrm{~g}$ to $5 \mathrm{~g}$ and maintained at this level for the rest of hospital stay. The patient was followed in hospital for a period of four weeks with no problems. After this time period, the patient was transferred to a skilled nursing facility for continuation of empiric IV antibiotics and $5 \mathrm{~g}$ of MMT for outpatient treatment.

\section{DISCUSSION}

The most significant cause of non-obstetric maternal death during pregnancy are diseases of the heart accounting for $10 \%$ of mortality during gestation [1]. Literature of infective endocarditis is an uncommon occurrence in obstetric and gynecological patients although it is being reported in greater incidence.

The use of heroin and benzodiazepines are associated with complications commonly following intravenous administration and the associated lifestyle that it leads. Needle use can cause localized infection including cellulitis, phlebitis, and superficial thrombosis, with systemic sequelae including endocarditis, septic osteomyelitis, and septicemia [2]. Opioid abuse during 
gestation is associated with multiple adverse outcomes for both the fetus and mother including intrauterine growth restriction, placental abruption, preterm births, risk of fetal demise, and neonatal abstinence syndrome (NAS) [5]. There is also increased risk for partaking in high-risk behaviors to support the addiction, which may place the woman at risk for acquiring sexually transmitted diseases, and becoming the victim of violence and legal ramifications [6].

Medication therapy is the standard of care to effectively manage addiction in the gravid patient. Since its use in the 1970's, methadone has been the first line therapy for gravidus women as it achieves the goal of stopping uncontrolled narcotic withdrawal and the associated secondary adverse outcomes [7]. Administered in a controlled setting once a day, methadone is effective at suppressing maternal cravings due to its long half-life. Based upon the relative efficacy and safety compared to other opioid medications, a 2012 report by the American Academy of Pediatrics (AAP) clinical report suggests that either morphine or methadone is the preferred opioid to prevent and treat NAS [8].

Right sided inflammation of the endocardial tissue and valves can be from IV derived infectious organisms which is due to the anatomical relevance of venous flow to the heart. Prior to the availability of antimicrobial therapy, infective endocarditis (IE) was invariably fatal. Although approximately $80 \%$ of patients now survive these infections, one in every six do not survive initial hospitalization, with up to one-third of patients infected with highly virulent organisms (such as Staphylococcus species) dying as direct or indirect result of valvular infection [9]. In contemporary times, IE presents more frequently as an acute disease rather than chronic state. Pertinent investigations combine thorough exploration of history and physical, echocardiography, blood cultures and other select laboratory studies to lead to an expeditious diagnosis [10].

A subset of IE patients present with serological studies that fail to identify causative organisms, known as culture negative endocarditis (CNE). Subacute presentations of endocarditis may present with leukocyte counts at normal or elevated values in patients [11]. Echocardiography should be performed on all patients with a moderate or high suspicion of endocarditis, this allows direct detection and characterization of vegetations on valves or other sites.

Treatment options exclusively include bactericidal categories for effective treatment in IE, avoiding bacteriostatic agents which only hinder growth. In general, therapy should target the organism isolated from blood cultures. For subacute presentation without causative microbial agent, standard of care dictates the administration of empirical therapy. Specific antibiotic regiments will not be discussed, but in general empirical care should include antibiotics for minimum of four to six weeks covering gram negative and gram positive bacteria [12].

\section{CONCLUSION}

This case reports an incidence of culture negative endocarditis (CNE) in obstetric practice derived from intravenous heroin abuse and how important multidiscipline collaboration is necessary in the clinical management of CNE in the gravid patient. This case highlights an important pathology that is part of the spectrum of disease being reported in greater incidence and a cause of significant mortality in obstetric patients. This presentation and review of appropriate diagnosis and management is beneficial for the education of physicians for this disease.

$$
* * * * * * * * *
$$

\section{Acknowledgements}

We wish to thank Mary Romero, MD (OB/GYN Hospitalist at Phoenix Perinatal Associates) for her contribution in patient care and acquisition of data.

\section{Author Contributions}

Alexander Sabre - Substantial contributions to conception and design, Acquisition of data, Drafting the article, Revising it critically for important intellectual content, Final approval of the version to be published Lydia Ehlenberger - Analysis and interpretation of data, Drafting the article, Final approval of the version to be published

\section{Guarantor}

The corresponding author is the guarantor of submission.

\section{Conflict of Interest}

Authors declare no conflict of interest.

\section{Copyright}

(C) 2015 Alexander Sabre et al. This article is distributed under the terms of Creative Commons Attribution License which permits unrestricted use, distribution and reproduction in any medium provided the original author(s) and original publisher are properly credited. Please see the copyright policy on the journal website for more information.

\section{REFERENCES}

1. Montoya ME, Karnath BM, Ahmad M. Endocarditis during Pregnancy. South Med J 2003 Nov;96(11):1156-7.

2. Llewelyn RW. Substance abuse in pregnancy: The team approach to antenatal care. The Obstetrician \& Gynaecologist 2000 Jan;2(1).

3. Stewart RD, Nelson DB, Adhikari EH, et al. The obstetrical and neonatal impact of maternal opioid detoxification in pregnancy. Am J Obstet Gynecol 2013 Sep;209(3):267.e1-5. 
4. Kandall SR, Albin S, Gartner LM, Lee KS, Eidelman A, Lowinson J. The narcotic-dependent mother: Fetal and neonatal consequences. Early Hum Dev 1977 Oct;1(2):159-69.

5. Kaltenbach K, Berghella V, Finnegan L. Opioid dependence during pregnancy. Effects and management. Obstet Gynecol Clin North Am 1998 Mar;25(1):139-51.

6. ACOG Committee Opinion No. 524: Opioid abuse, dependence, and addiction in pregnancy. Obstet Gynecol 2012 May;119(5):1070-6.

7. Patrick SW, Schumacher RE, Benneyworth BD, Krans EE, McAllister JM, Davis MM. Neonatal abstinence syndrome and associated health care expenditures: United States, 2000-2009. JAMA 2012 May 9;307(18):1934-40.
8. Herzlinger RA, Kandall SR, Vaughan HG Jr. Neonatal seizures associated with narcotic withdrawal. J Pediatr 1977 Oct;91(4):638-41.

9. Hudak ML, Tan RC. Neonatal drug withdrawal. Pediatrics 2012 Feb;129(2):e540-6o.

10. Fowler VG Jr, Miro JM, Hoen B, et al. Staphylococcus aureus endocarditis: A consequence of medical progress. JAMA 2005 Jun 22;293(24):3012-1.

11. Durack DT, Lukes AS, Bright DK. New Criteria for diagnosis of infective endocarditis: utilization of specific echocardiograhpic findings. Duke Endocarditis Service. Am J Med 1994 Mar;96(3):2009.

12. Sexton DJ, Calderwood SB, Baron EL. Antimicrobial therapy of native valve endocarditis. Wolter Kluwer Health. UpToDate 2014.

\section{ABOUT THE AUTHORS}

Article citation: Sabre A, Ehlenberger L. Acute opioid detoxification revealing a unique challenge in clinical management. Case Rep Int 2015;4:1-5.

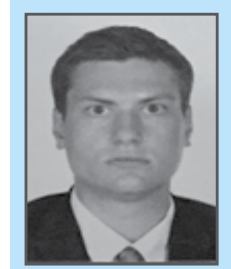

Alexander Sabre is 4th year Medical Student at Affiliated Hospital Scottsdale Healthcare System, Universidad Autonoma de Guadalajara, Guadalajara, Mexico. He earned undergraduate degree (B.S. Biology CMGB) from Drexel University, Philadelpiha, USA and postgraduate degree (Medico Cirujano) from (Universidad Autonoma de Guadalajara, Guadalajara, Mexico). He has published three research papers in national and international academic journals. His research interests include management of obstetric patients, maternal lactation research, importance of preconception follow-up in high-risk pregnancies, endocarditis in the gravid patient, fever of unknown origin, west Nile encephalitis. He intends to pursue a residency in $\mathrm{OB}$ and GYN in a program that will provide a solid foundation in general principles that will be important to pursue a fellowship in the field) in the future.

E-mail: alexandermsabre@gmail.com

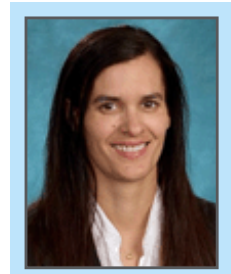

Lydia Ehlenberger is (MD. FACOG) Phoenix Perinatal Associates; OB Hospitalist; Obstetrix, Mednax; Phoenix, AZ, USA. Medical Degree earned at University of Arizona College of Medicine, Tucson, AZ. Board Certified, American Board of Obstetricians \& Gynecologists (ABOG). Fellow American College of Obstetricians \& Gynecologists (FACOG). Medical Acupuncture for Physician: UCLA Certification. Her research interests include (acupuncture in obstetric patients and congenital anomalies. Her medical mission continues to foster compassion for patients whilst achieving excellence in the high risk OB and GYN specialty. Her future interests include research in Acupunture treatments for the high risk antepartum Obstetric patient. Having recently completed "Medical acupuncture for Physicians:" UCLA certified, She intend to maintain her certification with hopes to create an academic environment in blending evidence based medicine with the daily clinical practice in our high risk antepartum unit, at best we can bridge the gap between the historical paradigms of medicine.

E-mail: lydia_ehlenberger@Obstetrix.com 
Access full text article on other devices

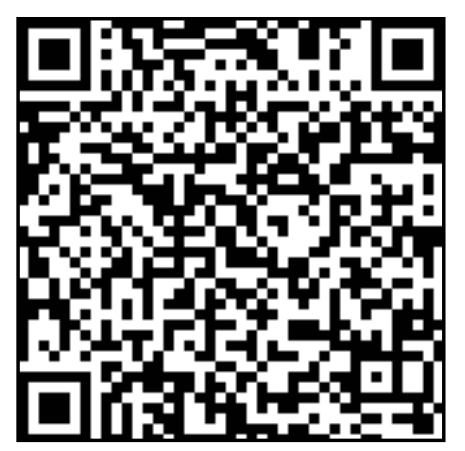

Access PDF of article on other devices

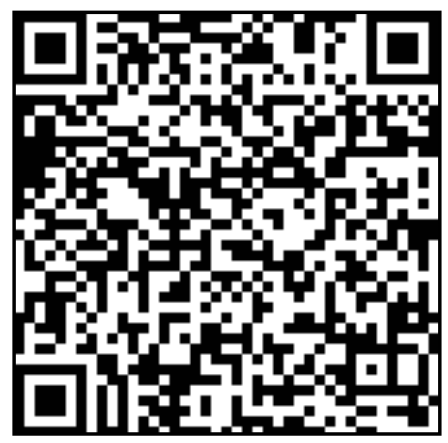

